Pacific Journal of Mathematics

GLOBAL PROPERTIES OF RATIONAL AND
LOGARITHMICO-RATIONAL MINIMAL SURFACES 


\section{GLOBAL PROPERTIES OF RATIONAL AND LOGARITHMICO-RATIONAL MINIMAL SURFACES}

E. F. Beckenbach, F. H. Eng, and R. E. TAFel

In this paper, rational and logarithmico-rational minimal surfaces are defined and some of their properties are investigated. In particular, it is shown-perhaps somewhat surprisingly-that the fundamental theorem of algebra, suitably formulated, holds for these surfaces.

1. Introduction. Let $f(w)$ be a rational complex function, of degree $m$, of the complex variable $w$; that is, let $f(w)$ admit a representation of the form

$$
f(w)=\frac{p(w)}{q(w)},
$$

where $p(w)$ and $q(w)$ are relatively prime complex polynomial functions:

$$
[p(w), q(w)]=1
$$

and let

$$
\operatorname{deg} f(w)=\max [\operatorname{deg} p(w), \operatorname{deg} q(w)]=m .
$$

It is well known (see, for instance, [1, p. 31]) that, with multiple values suitably counted, for any such rational function of degree $m$, $m \geqq 1$, the equation $\zeta=f(w)$ maps the closed single-sheeted $w$-plane onto the closed $m$-sheeted $\zeta$-plane. That is, each $\zeta$-value, including $\infty$, is taken on exactly $m$ times (counting multiplicities) as $w$ ranges once over the closed complex plane. This is a rather immediate consequence of the fundamental theorem of algebra.

Now for $n \geqq 3$, a (two-dimensional) minimal surface $S$ in $n$-dimensional Euclidean space $E_{n}$ has $n$-dimensional measure 0 , and accordingly the foregoing result concerning complex rational functions $f(w)$ could hardly be expected to extend directly to minimal-surface theory. It is nevertheless one purpose of this paper to show (see §10) that the result, when suitably formulated, does indeed extend precisely to minimal surfaces.

Since, as we shall see in $\S 4$, the map $\zeta=f(w)$ given by a rational complex function $f(w)$ is a special rational minimal surface, it therefore follows in particular that the fundamental theorem of algebra concerning plane maps can be given a formulation relative to the points of any containing $n$-dimensional Euclidean space! 
2. Meromorphic minimal surfaces. A surface $S$ in $n$-dimensional Euclidean space $E_{n}, n \geqq 2$, is said to be given in terms of isothermal parameters $u, v$ provided the representation

$$
S: x_{j}=x_{j}(u, v), \quad j=1,2, \cdots, n,
$$

or

$$
S: \boldsymbol{x}=\boldsymbol{x}(u, v),
$$

for $(u, v) \in D$, where $D$ is some finite domain of definition, is such that

$$
E=G=\lambda(u, v), \quad F=0,
$$

where

$$
E=\frac{\partial \boldsymbol{x}}{\partial u} \cdot \frac{\partial \boldsymbol{x}}{\partial u}, F=\frac{\partial \boldsymbol{x}}{\partial u} \cdot \frac{\partial \boldsymbol{x}}{\partial v}, G=\frac{\partial \boldsymbol{x}}{\partial v} \cdot \frac{\partial \boldsymbol{x}}{\partial v} .
$$

Such an isothermal representation is conformal, or angle-preserving, except at points where $\lambda(u, v)=0$.

According to a theorem of Weierstrass [16, p. 27], a necessary and sufficient condition that the surface (1), given in terms of isothermal parameters, be a minimal surface is that the vector function $\boldsymbol{x}(u, v)$ be harmonic:

$$
\Delta \boldsymbol{x}(u, v) \equiv \frac{\partial^{2} \boldsymbol{x}}{\partial u^{2}}+\frac{\partial^{2} \boldsymbol{x}}{\partial v^{2}}=0 .
$$

Then in any simply connected part of $D$, the functions $x_{j}(u, v)$ are the real parts of analytic functions of a complex variable,

$$
x_{j}(u, v)=\Re f_{j}(w), \quad w=u+i v,
$$

and (2) is equivalent to

$$
\sum_{j=1}^{n}\left[\frac{d f_{j}(w)}{d w}\right]^{2}=0
$$

If an isothermal representation (1) of the minimal surface $S$ is such that all the coordinate functions except two are identically zero, say $x_{j}(u, v) \equiv 0, j=3,4, \cdots, n$, then either $x_{1}(u, v)+i x_{2}(u, v)$ or $x_{2}(u$, $v)+i x_{1}(u, v)$ is an analytic function of the complex variable $w=u+$ $i v$, and $x_{1}(u, v)$ and $x_{2}(u, v)$ are said to be a couple of conjugate harmonic functions. By analogy, the coordinate functions of any minimal surface $S$ in $E_{n}$, given in isothermal representation, are called an n-tuple of conjugate harmonic functions [8].

For a given $\left(u_{0}, v_{0}\right)$, let

$$
u-u_{0}=r \cos \theta, \quad v-v_{0}=r \sin \theta,
$$


and suppose that for some $R_{1}, R_{2}$ the minimal surface $S$ is given in isothermal representation by (1) in the annulus

$$
R_{1}<r<R_{2} \text {. }
$$

Since the vector function $\boldsymbol{x}(u, v)$ is harmonic in (5), it can be represented [14, p. 692] there by a series of the form

$$
\boldsymbol{x}(u, v)=\boldsymbol{c} \log r+\sum_{k=-\infty}^{\infty} r^{k}\left(\boldsymbol{a}_{k} \cos k \theta+\boldsymbol{b}_{k} \sin k \theta\right) .
$$

The constant vector $\boldsymbol{b}_{0}$ is arbitrary; we shall henceforth assume that

$$
b_{0}=\mathbf{0} \text {. }
$$

The other coefficients, including the logarithmic coefficient $\boldsymbol{c}$, are uniquely determined by $\boldsymbol{x}(u, v)$.

The harmonic vector function $\boldsymbol{x}(u, v)$ given by (6) also satisfies the condition (2), since $S$ is given in isothermal representation. For the representation (6), the condition (2) are equivalent $[4,5,6,7]$ to

$$
\left\{\begin{array}{l}
k \boldsymbol{b}_{k} \cdot \boldsymbol{c}+\sum_{l=-\infty}^{\infty} l(k-l) \boldsymbol{a}_{l} \cdot \boldsymbol{b}_{k-l}=0, k=0, \pm 1, \pm 2, \cdots \\
2 k \boldsymbol{a}_{k} \cdot \boldsymbol{c}+\sum_{l=-\infty}^{\infty} l(k-l)\left(\boldsymbol{a}_{l} \cdot \boldsymbol{a}_{k-l}-\boldsymbol{b}_{l} \cdot \boldsymbol{b}_{k-l}\right)=0, k= \pm 1, \pm 2, \cdots \\
\boldsymbol{c} \cdot \boldsymbol{c}-2 \sum_{l=1}^{\infty} l^{2}\left(\boldsymbol{a}_{l} \cdot \boldsymbol{a}_{-l}-\boldsymbol{b}_{l} \cdot \boldsymbol{b}_{-l}\right)-0 .
\end{array}\right.
$$

If $R_{1}=0$ in (5), we say that $S$ has an isolated singularity at $\left(u_{0}, v_{0}\right)$. We then call

$$
\mathscr{P}_{0}[\boldsymbol{x}(u, v)]=\sum_{k=-\infty}^{-1} \boldsymbol{r}^{k}\left(\boldsymbol{a}_{k} \cos k \theta+\boldsymbol{b}_{k} \sin k \theta\right)
$$

the principal part (cf. [11, pp. 212, 213]), and

$$
\mathscr{L}_{0}[\boldsymbol{x}(u, v)]=\boldsymbol{c} \log r=\boldsymbol{c} \log \left|w-w_{0}\right|, w_{0}=u_{0}+i v_{0},
$$

the logarithmic part, of (6).

Similarly, if $R_{2}=\infty$ in (5) we say that $S$ has an isolated singularity at $w=\infty$. The transformation

$$
\begin{aligned}
W=U+i V & =\frac{1}{w-w_{0}}=\frac{1}{\left(u-u_{0}\right)+i\left(v-v_{0}\right)} \\
& =\frac{\left(u-u_{0}\right)-i\left(v-v_{0}\right)}{\left(u-u_{0}\right)^{2}+\left(v-v_{0}\right)^{2}}=\frac{1}{r} \cos \theta-\frac{i}{r} \sin \theta
\end{aligned}
$$

then maps (5) conformally onto 


$$
0<|W|<1 / R_{1}
$$

and the isolated singularity at $w=\infty$ is treated by discussing it as an isolated singularity at $W=0$. The principal part of (6) now becomes

$$
\mathscr{P}_{\infty}\left[\boldsymbol{x}(u, v) ; w_{0}\right]=\sum_{k=1}^{\infty} \boldsymbol{r}^{k}\left(\boldsymbol{a}_{k} \cos k \theta+\boldsymbol{b}_{k} \sin k \theta\right),
$$

and the logarithmic part becomes

$$
\mathscr{L}_{\infty}\left[\boldsymbol{x}(u, v) ; w_{0}\right]=\boldsymbol{c} \log r=\boldsymbol{c} \log \left|w-w_{0}\right| .
$$

The sum of $\boldsymbol{x}\left(u_{0}, v_{0}\right)$ and the principal part $\mathscr{P}_{\infty}\left[\boldsymbol{x}(u, v) ; w_{0}\right]$, and likewise the logarithmic coefficient $\boldsymbol{c}$ in the logarithmic part $\mathscr{L}_{\infty}[\boldsymbol{x}(u$, $\left.v) ; w_{0}\right]$, are independent of $\left(u_{0}, v_{0}\right)$.

The equations (8) are more tractable than they might at first glance appear to be.

For $n=2$, the equations (8) reduce simply to

$$
b_{1, k}= \pm a_{2, k}, b_{2, k}=\mp a_{1, k}, c_{1}=c_{2}=0, k= \pm 1, \pm 2, \cdots \text {. }
$$

Thus if $n=2$ then the series for $x_{2}(u, v)$ in (6) can be written, to within sign and the arbitrary additive constant $a_{2,0}$, directly from the series for $x_{1}(u, v)$; and neither of the two series can contain a logarithmic term.

Let the minimal surface $S$ in $E_{n}$ be given in isothermal representation by (6) for $0<r<R$, so that $S$ has an isolated singularity at $\left(u_{0}, v_{0}\right)$. We distinguish four cases, $(\mathrm{a}),(\mathrm{b}),\left(\mathrm{c}^{\prime}\right)$, and $\left(\mathrm{c}^{\prime \prime}\right)$ :

(a) Essential singularity. If for an infinitude of negative indices $l$ we have

$$
\boldsymbol{a}_{l} \cdot \boldsymbol{a}_{l}+\boldsymbol{b}_{l} \cdot \boldsymbol{b}_{l} \neq 0,
$$

then we say that the isolated singularity of $S$ at $\left(u_{0}, v_{0}\right)$ is essential; otherwise, we say that the isolated singularity is nonessential.

(b) Pole. If $S$ has a nonessential isolated singularity at $\left(u_{0}, v_{0}\right)$, and the lowest index $l=t$ for which (14) holds is negative, then we say that $S$ has a pole of order $|t|$ at $\left(u_{0}, v_{0}\right)$. By definition, then, the poles of $S$ are isolated.

If $S$ has a pole of order $-t>0$ at $\left(u_{0}, v_{0}\right)$, then (9) reduces to

$$
\begin{aligned}
\mathscr{P}_{0}[\boldsymbol{x}(u, v)] & =\sum_{k=t}^{-1} \boldsymbol{r}^{k}\left(\boldsymbol{a}_{k} \cos k \theta+\boldsymbol{b}_{k} \sin k \theta\right) \\
& =\Re \sum_{k=t}^{-1}\left(\boldsymbol{a}_{k}-i \boldsymbol{b}_{k}\right)\left(w-w_{0}\right)^{k} \\
& =\Re \frac{\boldsymbol{p}(w)}{q(w)},
\end{aligned}
$$

where $\boldsymbol{p}(w)$ is a complex polynomial vector function of $w$, of degree 
less than $|t| ; q(w)=\left(w-w_{0}\right)^{|t|}$ is a complex polynomial scalar function of $w ; \boldsymbol{p}(w)$ and $q(w)$ are relatively prime:

$$
[p(w), q(w)]=1
$$

and

$$
\operatorname{deg} \frac{p(w)}{q(w)}=\max [\operatorname{deg} \boldsymbol{p}(w), \operatorname{deg} q(w)]=|t| .
$$

If (14) does not hold for any $l<0$, so that

$$
\mathscr{P}_{0}[\boldsymbol{x}(u, v)] \equiv \mathbf{0},
$$

then it follows from the third equation in (8) that

$$
c \cdot c=0,
$$

and therefore that

$$
\mathscr{L}_{0}[\boldsymbol{x}(u, v)] \equiv \mathbf{0}
$$

Hence a minimal surface given in isothermal representation by (1) cannot have an isolated singularity that is merely logarithmic. [But see example (e) in §5.]

(c) Removable singularity. If (14) does not hold for any $l<0$, then we say that $S$ has a removable singularity at $\left(u_{0}, v_{0}\right)$. In this case, we adjoin to $S$ the point $\boldsymbol{a}_{0}$ corresponding to $\left(u_{0}, v_{0}\right)$, if indeed this correspondence was not already given in the definition of $S$. Then the vector function (6) gives an isothermal map of $|w|<R$ onto the (extended) surface, which we again denote by $S$. We then say that $S$ is regular at $\left(u_{0}, v_{0}\right)$.

If $S$ is regular at $\left(u_{0}, v_{0}\right)$, then either

$\left(c^{\prime}\right) \quad \boldsymbol{x}(u, v) \equiv \boldsymbol{a}_{0}$ and $S$ reduces to a point, or

$\left(\mathrm{c}^{\prime \prime}\right)$ there is a lowest positive index $l=t$ for which (14) holds. In the former case $\left(c^{\prime}\right)$, we say that $S$ is a constant minimal surface. In the latter case $\left(\mathrm{c}^{\prime \prime}\right)$, we say that $S$ has an $\boldsymbol{a}_{0}$-point of order $t$ at $\left(u_{0}, v_{0}\right)$; in particular, if $\boldsymbol{a}_{0}=\mathbf{0}$ then we say that $S$ has a zero of order $t$ at $\left(u_{0}, v_{0}\right)$.

If $S$ has a pole of order $-t>0$ or an $a_{0}$-point of order $t>0$ at $\left(u_{0}, v_{0}\right)$, then for $k=2 t$ the first two equations in (8) reduce respectively to

$$
t^{2} \boldsymbol{a}_{t} \cdot \boldsymbol{b}_{t}=0 \text { and } t^{2}\left(\boldsymbol{a}_{t} \cdot \boldsymbol{a}_{t}-\boldsymbol{b}_{t} \cdot \boldsymbol{b}_{t}\right)=0 \text {, }
$$

whence

$$
\boldsymbol{a}_{t} \cdot \boldsymbol{b}_{t}=0 \text { and } \boldsymbol{a}_{t} \cdot \boldsymbol{a}_{t}=\boldsymbol{b}_{t} \cdot \boldsymbol{b}_{t} \neq 0 \text {. }
$$

If $S$ has a pole of order $-t>0$ at $\left(u_{0}, v_{0}\right)$, then from (6) and 
(17) we obtain

$$
\boldsymbol{x}(u, v) \cdot \boldsymbol{x}(u, v)=r^{2 t} \boldsymbol{a}_{t} \cdot \boldsymbol{a}_{t}+o\left(r^{2 t}\right)
$$

as $r \rightarrow 0$. Similarly, if $S$ has an $\boldsymbol{a}_{0}$-point of order $t>0$ at $\left(u_{0}, v_{0}\right)$, then

$$
\left[\boldsymbol{x}(u, v)-\boldsymbol{a}_{0}\right] \cdot\left[\boldsymbol{x}(u, v)-\boldsymbol{a}_{0}\right]=r^{2 t} \boldsymbol{a}_{t} \cdot \boldsymbol{a}_{t}+o\left(r^{2 t}\right) .
$$

By (18) and (19) we thus see that if $S$ does not reduce to a point, then not only the poles but also the finite a-points of $S$ are isolated [6].

Again if $S$ has a pole of order $-t>0$ or an $\boldsymbol{a}_{0}$-point of order $t>0$ at $\left(u_{0}, v_{0}\right)$, then from (2), (6), and (17) we obtain

$$
\lambda(u, v)=t^{2} r^{2 t-2} \boldsymbol{a}_{t} \cdot \boldsymbol{a}_{t}+O\left(r^{2 t-1}\right)
$$

as $r \rightarrow 0$. Therefore, if $t<0$ then $\lambda(u, v)$ has an isolated infinite value at $\left(u_{0}, v_{0}\right)$; if $t=1$ then $\lambda(u, v)$ has a finite nonzero value there; and if $t>1$ then $\lambda(u, v)$ has an isolated zero at $\left(u_{0}, v_{0}\right)$. Thus if $S$ is a nonconstant minimal surface, and $S$ has no singularities other than poles, then the zeros and infinities of $\lambda(u, v)$ are isolated.

For the present theory, we extend Euclidean $n$-space by postulating a single ideal point at $\infty$. In this space, the isothermal transformation

$$
x^{*}=\frac{x}{x \cdot x}
$$

effects an inversion [10] in the unit hypersphere with center at the origin. If $S$ has a pole, or $\infty$-point, of order $-t>0$ at $\left(u_{0}, v_{0}\right)$, then the surface

$$
S^{*}: \boldsymbol{x}=\boldsymbol{x}^{*}(u, v)=\frac{\boldsymbol{x}(u, v)}{\boldsymbol{x}(u, v) \cdot \boldsymbol{x}(u, v)}
$$

has a zero of order $|t|$ at $\left(u_{0}, v_{0}\right)$. The surface $S^{*}$ will not ordinarily be a minimal surface, but since the transformation is isothermal we say that the measure of the angle between arcs from the origin on $S^{*}$ is the measure of the angle between corresponding ares from the point at $\infty$ on $S$.

If $S$ has a pole of order $-t>0$ at $\left(u_{0}, v_{0}\right)$, then (17) and (18) show, through a cosine evaluation, that measures of angles between ares from $\left(u_{0}, v_{0}\right)$ are multiplied by $|t|$ in the map onto $S^{*}$ and therefore, by definition, in the map onto $S$.

Similarly, if $S$ has an $\boldsymbol{a}_{0}$-point of order $t>0$ at $\left(u_{0}, v_{0}\right)$ then again (17) and (18) show, through a cosine evaluation, that measures of angles between arcs from $\left(u_{0}, v_{0}\right)$ are multiplied by $t$ in the map onto $S$. If, except for poles, $S$ is a regular minimal surface given in 
isothermal representation by $(1)$ for $(u, v)$ in a domain $D$, then we say that $S$ is a meromorphic minimal surface for $(u, v)$ in $D$. In particular, if $D$ is the entire finite plane then we simply say that $S$ is a meromorphic minimal surface. In the latter case, if $S$ has no poles in the finite plane then we say that $S$ is an entire minimal surface.

3. Rational and logarithmico-rational minimal surfaces. Let the meromorphic minimal surface $S$ in $E_{n}$, given in isothermal representation by (1), have at most a finite number of poles, at the points $w_{1}, w_{2}, \cdots, w_{l}, l \geqq 0$, in the finite $w$-plane, $w=u+i v$; let the orders of these poles be $n_{1}, n_{2}, \cdots, n_{l}$, respectively; and let the corresponding logarithmic coefficients be $c_{1}, c_{2}, \cdots, c_{l}$.

Then, by (15), the principal part of $\boldsymbol{x}(u, v)$ at $w_{j}, j=1,2, \cdots, l$, is

$$
\mathscr{P}_{j}[\boldsymbol{x}(u, v)]=\mathfrak{R} \frac{\boldsymbol{p}_{j}(w)}{q_{j}(w)},
$$

where $\boldsymbol{p}_{j}(w)$ is a polynomial vector function of degree less than $n_{j}$, $q_{j}(w)=\left(w-w_{j}\right)^{n_{j}}$, and $\boldsymbol{p}_{j}(w)$ and $q_{j}(w)$ are relatively prime. The logarithmic part of $\boldsymbol{x}(u, v)$ at $w_{j}$ is

$$
\mathscr{L}_{j}[\boldsymbol{x}(u, v)]=\boldsymbol{c}_{j} \log \left|w-w_{j}\right| .
$$

Now the vector function

$$
\boldsymbol{y}(u, v)=\boldsymbol{x}(u, v)-\sum_{j=1}^{l} \mathscr{P}_{j}[\boldsymbol{x}(u, v)]-\sum_{j=1}^{l} \mathscr{L}_{j}[\boldsymbol{x}(u, v)]
$$

is harmonic in the entire finite plane, since each term in the right-hand member is harmonic except at the points $w_{j}$, and since the singularities at the $w_{j}$ exactly cancel out. Therefore, $\boldsymbol{y}(u, v)$ can be represented in the finite plane by a series of the form

$$
\begin{aligned}
\boldsymbol{y}(u, v) & =\boldsymbol{y}(r \cos \theta, r \sin \theta) \\
& =\sum_{k=0}^{\infty} r^{k}\left(\boldsymbol{a}_{k} \cos k \theta+\boldsymbol{b}_{k} \sin k \theta\right) .
\end{aligned}
$$

Thus from (23) and (24) we have

$$
\begin{aligned}
\boldsymbol{x}(u, v)= & \sum_{j=1}^{l} \mathscr{P}_{j}[\boldsymbol{x}(u, v)]+\sum_{j=1}^{l} \mathscr{L}_{\jmath}[\boldsymbol{x}(u, v)] \\
& +\sum_{k=0}^{\infty} r^{k}\left(\boldsymbol{a}_{k} \cos k \theta+\boldsymbol{b}_{k} \sin k \theta\right) .
\end{aligned}
$$

In particular, let the minimal surface $S$ in $E_{n}$, given in isothermal representation by (1), be meromorphic in the closed $w$-plane, $w=$ $u+i v$.

Then, since poles are isolated, $S$ can have at most a finite number of poles in all, and accordingly the vector function $\boldsymbol{x}(u, v)$ has 
a representation of the form (25).

But now $\boldsymbol{x}(u, v)$ has at most a pole at $w=\infty$, and therefore, for some integer $n_{\infty} \geqq 0$,

$$
\begin{aligned}
\boldsymbol{x}(u, v)=\boldsymbol{a}_{0} & +\sum_{j=1}^{l} \mathscr{P}_{j}[\boldsymbol{x}(u, v)]+\sum_{k=1}^{n_{\infty}} \boldsymbol{r}^{k}\left(\boldsymbol{a}_{k} \cos k \theta+\boldsymbol{b}_{k} \sin k \theta\right) \\
& +\sum_{j=1}^{l} \mathscr{L}_{j}[\boldsymbol{x}(u, v)] \\
= & \boldsymbol{a}_{0}+\sum_{j=1}^{l} \mathscr{P}_{j}[\boldsymbol{x}(u, v)]+\mathscr{P}_{\infty}[\boldsymbol{x}(u, v) ; 0]+\sum_{j=1}^{l} \mathscr{L}_{j}[\boldsymbol{x}(u, v)] .
\end{aligned}
$$

Since, in (26), $\mathscr{P}_{\infty}[x(u, v) ; 0]$ is the 0 polynomial vector function if $n_{\infty}=0$, and is a polynomial vector function of degree $n_{\infty}$ if $n_{\infty}>0$, it follows from (21) that

$$
\boldsymbol{a}_{0}+\sum_{j=1}^{l} \mathscr{P}_{j}[\boldsymbol{x}(u, v)]+\mathscr{P}_{\infty}[\boldsymbol{x}(u, v) ; 0]=\mathscr{P}[\boldsymbol{x}(u, v)]=\mathfrak{R} \frac{\boldsymbol{p}(w)}{q(w)}
$$

where $\boldsymbol{p}(w)$ is a polynomial vector function;

$$
q(w)=\prod_{j=1}^{l}\left(w-w_{j}\right)^{n_{j}} ;
$$

$\boldsymbol{p}(w)$ and $q(w)$ are relatively prime; and

$$
\operatorname{deg} \frac{\boldsymbol{p}(w)}{q(w)}=\max [\operatorname{deg} \boldsymbol{p}(w), \operatorname{deg} q(w)]=n_{\infty}+\sum_{j=1}^{l} n_{j}
$$

By (22),

$$
\begin{aligned}
\sum_{j=1}^{l} \mathscr{L}_{j}[\boldsymbol{x}(u, v)] & =\mathscr{L}[\boldsymbol{x}(u, v)] \\
& =\sum_{j=1}^{l} \boldsymbol{c}_{j} \log \left|w-w_{j}\right|=\log \prod_{j=1}^{l}\left|w-w_{j}\right|^{\left.\right|_{j}} .
\end{aligned}
$$

It follows from (27) and (29) that (26) can be written as

$$
\begin{aligned}
\boldsymbol{x}(u, v) & =\mathscr{P}[\boldsymbol{x}(u, v)]+\mathscr{L}[\boldsymbol{x}(u, v)] \\
& =\Re \frac{\boldsymbol{p}(w)}{q(w)}+\log \prod_{j=1}^{l}\left|w-w_{j}\right|^{c_{j}} .
\end{aligned}
$$

If $c_{j}=\mathbf{0}$ for all $j, j=1,2, \cdots, l$, then (30) reduces to

$$
\boldsymbol{x}(u, v)=\mathscr{P}[\boldsymbol{x}(u, v)]=\mathfrak{R} \frac{\boldsymbol{p}(w)}{q(w)},
$$

and we say that $S$ is a rational minimal surface of degree

$$
m=n_{\infty}+\sum_{j=1}^{l} n_{j}
$$


[or is the zero rational minimal surface if $\boldsymbol{x}(u, v)=\mathbf{0}$ ].

If the $c_{j}, j=1,2, \cdots, l$, are not all 0 , then we say that $S$, given by (30), is a logarithmico-rational minimal surface of degree $m$, where $m$ is given by (32).

In accordance with these definitions, if $S$ is a rational minimal surface, or a logarithmico-rational minimal surface, of degree $m$, then $S$ has exactly $m$ poles, counting multiplicities, in the closed w-plane.

We have thus shown that if a minimal surface $S$, given in isothermal representation, is meromorphic in the closed plane, then $S$ is either a rational minimal surface or a logarithmico-rational minimal surface.

Conversely, if a minimal surface $S$, given in isothermal representation, is either a rational minimal surface or a logarithmico-rational minimal surface, then $S$ clearly is meromorphic in the closed plane.

We accordingly have the following result.

THEOREM 1. A minimal surface $S$, given in isothermal representation, is meromorphic in the closed plane if and only if $S$ is either a rational minimal surface or a logarithmico-rational minimal surface.

4. Remarks. (a) For a logarithmico-rational minimal surface $S$, given in isothermal representation by (1), the vector $c_{j}$ in (30) might be called the order of the logarithmic part of $\boldsymbol{x}(u, v)$ at the singular point $w=w_{j}$. Since

$$
\lim _{w \rightarrow \infty}\left|\frac{w-w_{j}}{w}\right|=1,
$$

by (12) and (30) we have

$$
\begin{aligned}
\mathscr{L}_{\infty}[\boldsymbol{x}(u, v) ; 0] & =\sum_{j=1}^{l} \boldsymbol{c}_{j} \log |w| \\
& =-\sum_{j=1}^{l} \boldsymbol{c}_{j} \log |W|, \quad W=\frac{1}{w} .
\end{aligned}
$$

We therefore take

$$
\boldsymbol{c}_{\infty}=-\sum_{j=1}^{l} \boldsymbol{c}_{j}
$$

as the order of the logarithmic part of $\boldsymbol{x}(u, v)$ at $w=\infty$.

Then for any logarithmico-rational minimal surface $S$, given in isothermal representation by (1), the sum of the orders of the logarithmic part of $\boldsymbol{x}(u, v)$ at all the singular points of $S$ is $\mathbf{0}$, since

$$
\sum_{j=1}^{l} \boldsymbol{c}_{j}+\boldsymbol{c}_{\infty}=\sum_{j=1}^{l} \boldsymbol{c}_{j}-\sum_{j=1}^{l} \boldsymbol{c}_{j}=\mathbf{0} .
$$


(b) By (13), in $E_{2}$ there are no logarithmico-rational minimal surfaces. [But see example (e) in §5.]

(c) Let $S$ be a rational or logarithmico-rational minimal surface in $E_{n}$, given in isothermal representation by (1), for which the function $q(w)$ in (30) satisfies $q(w) \equiv 1$. Then (14) does not hold for any $l<0$, and therefore, by the third equation in (8), $c$ satisfies (16). Thus $S$ must be a rational minimal surface. Since also $q(w) \equiv 1$, we say that $S$ is a polynomial, or entire rational, minimal surface. There are no logarithmico-polynomial minimal surfaces.

(d) If $S$ is a rational (or polynomial) minimal surface of degree $m$, given in isothermal representation by $(1)$, and $x_{j}(u, v) \equiv 0, j=3$, $4, \cdots, n$, then either $x_{1}(u, v)+i x_{2}(u, v)$ or $x_{2}(u, v)+i x_{1}(u, v)$ is a rational (or polynomial) complex function, of degree $m$, of the complex variable $w=u+i v$. Thus the study of rational (or polynomial) minimal surfaces of degree $m$ subsumes the study of rational (or polynomial) complex functions of degree $m$.

(e) We note that the expression $\Re[p(w) / q(w)]$ in (27) can be written as a rational harmonic vector function of the real variables $u, v$, since

$$
\Re \frac{\boldsymbol{p}(w)}{q(w)}=\Re \frac{\boldsymbol{p}(w) \overline{q(w)}}{q(w) \overline{q(w)}}=\frac{\Re \boldsymbol{p}(w) \overline{q(w)}}{q(w) \overline{q(w)}}=\frac{\boldsymbol{P}(u, v)}{Q(u, v)},
$$

and $\boldsymbol{P}(u, v)$ and $Q(u, v)$ are polynomial functions of $u, v$, as desired.

Although $P(u, v) / Q(u, v)$ is a harmonic vector function of $u, v$, the functions $\boldsymbol{P}(u, v)$ and $Q(u, v)$ themselves ordinarily are not harmonic.

By (28), (32), and (34), the degree $m$ of $S$ is given by

$$
m=\max [\operatorname{deg} P(u, v), \operatorname{deg} Q(u, v)]-\frac{1}{2} \operatorname{deg} Q(u, v) .
$$

(f) We note also, conversely, that if $\boldsymbol{x}(u, v)$ is a rational harmonic vector function,

$$
\boldsymbol{x}(u, v)=\frac{\boldsymbol{P}(u, v)}{Q(u, v)},
$$

where $\boldsymbol{P}(u, v)$ and $Q(u, v)$ are polynomial functions of $u, v$, and we let

$$
\boldsymbol{x}(w, v)=\Re f(w),
$$$$
w=u+i v,
$$

where $\boldsymbol{f}(w)$ is an analytic vector function of $w$, then $\boldsymbol{f}(w)$ is a rational vector function of $w$, since, in a neighborhood of any point $\left(u_{0}, v_{0}\right)$, or $w_{0}=u_{0}+i v_{0}$, at which $Q(u, v) \neq 0, f(w)$ is given (see, for instance, $[9$, p. 127]), to within an arbitrary additive pure imaginary constant vector, by 


$$
\begin{aligned}
\boldsymbol{f}(w) & =2 \boldsymbol{x}\left(\frac{w+\bar{w}_{0}}{2}, \frac{w-\bar{w}_{0}}{2 i}\right)-\boldsymbol{x}\left(u_{0}, v_{0}\right) \\
& =2 \frac{\boldsymbol{P}\left(\frac{w+\bar{w}_{0}}{2}, \frac{w-\bar{w}_{0}}{2 i}\right)}{Q\left(\frac{w+\bar{w}_{0}}{2}, \frac{w-\bar{w}_{0}}{2 i}\right)}-\frac{\boldsymbol{P}\left(u_{0}, v_{0}\right)}{Q\left(u_{0}, v_{0}\right)}=\frac{\boldsymbol{p}(w)}{q(w)},
\end{aligned}
$$

and $\boldsymbol{p}(w)$ and $q(w)$ are polynomial functions of $w$. By analytic continuation, $\boldsymbol{f}(w)$ is therefore a rational vector function of $w$ throughout its domain of analyticity.

5. Examples. (a) The minimal surface $S$ of Enneper [13, p. 221] is given in isothermal representation by

$$
\begin{aligned}
& x_{1}(u, v)=3 u-u^{3}+3 u v^{2}=\Re\left(3 w-w^{3}\right)=3 r \cos \theta-r^{3} \cos 3 \theta, \\
& x_{2}(u, v)=-3 v+v^{3}-3 u^{2} v=\Re\left(3 i w+i w^{3}\right)=-3 r \sin \theta-r^{3} \sin 3 \theta, \\
& x_{3}(u, v)=3 u^{2}-3 v^{2}=\Re\left(3 w^{2}\right)=3 r^{2} \cos 2 \theta .
\end{aligned}
$$

This surface is a polynomial, or entire rational, minimal surface of degree 3. In the closed $w$-plane, $S$ has just one zero; this is at $w=0$ and is of order 1. The surface has a pole of order 3 at $w=\infty$.

(b) The functions

$$
\begin{aligned}
& x_{1}(u, v)=\frac{\left(1+u^{2}+v^{2}\right) u}{u^{2}+v^{2}}=\Re\left(\frac{1}{w}+w\right)=\left(\frac{1}{r}+r\right) \cos \theta, \\
& x_{2}(u, v)=\frac{\left(1+u^{2}+v^{2}\right) v}{u^{2}+v^{2}}=\Re\left(\frac{i}{w}-i w\right)=\left(\frac{1}{r}+r\right) \sin \theta, \\
& x_{3}(u, v)=\log \left(u^{2}+v^{2}\right)=\Re(2 \log w)=2 \log r
\end{aligned}
$$

are the coordinate functions of a logarithmico-rational minimal surface (actually a catenoid) in isothermal representation. The surface is of degree 2 , with poles of order 1 at $w=0$ and at $w=\infty$; it has no zero.

(c) The rational minimal surfaces of Eng, given in isothermal Weierstrass-formula [16, pp. 28, 29] representation by

$$
\begin{aligned}
& x_{1}(u, v)=\Re f_{1}(w)=\Re \int^{w}\left(1-\zeta^{2}\right)\left[\frac{a}{(1-\zeta)^{4}}+\frac{b}{(i-\zeta)^{4}}+\frac{c}{\zeta^{4}}\right] d \zeta, \\
& x_{2}(u, v)=\Re f_{2}(w)=\Re \int^{w} i\left(1+\zeta^{2}\right)\left[\frac{a}{(1-\zeta)^{4}}+\frac{b}{(i-\zeta)^{4}}+\frac{c}{\zeta^{4}}\right] d \zeta, \\
& x_{3}(u, v)=\Re f_{3}(w)=\Re \int^{w} 2 \zeta\left[\frac{a}{(1-\zeta)^{4}}+\frac{b}{(i-\zeta)^{4}}+\frac{c}{\zeta^{4}}\right] d \zeta,
\end{aligned}
$$

where $a, b$, and $c$ are arbitrary nonzero real constants, are of degree 9 , with poles of order 3 at $w=0, w=1$, and $w=i$. Although the standard representation (30) of each of these surfaces is 


$$
\begin{aligned}
& x_{1}(u, v)=\Re f_{1}(w)=\Re \frac{(1-w) p_{1}(w)}{q(w)}, \\
& x_{2}(u, v)=\Re f_{2}(w)=\Re \frac{(i-w) p_{2}(w)}{q(w)}, \\
& x_{3}(u, v)=\Re f_{3}(w)=\Re \frac{w p_{3}(w)}{q(w)},
\end{aligned}
$$

where $q(w)=(1-w)^{3}(i-w)^{3} w^{3}$ is of degree 9 , each of the functions $f_{j}(w)$ actually is of degree only 8 :

$$
\begin{aligned}
& f_{1}(w)=\frac{p_{1}(w)}{q_{1}(w)}=\frac{p_{1}(w)}{(1-w)^{2}(i-w)^{3} w^{3}}, \\
& f_{2}(w)=\frac{p_{2}(w)}{q_{2}(w)}=\frac{p_{2}(w)}{(1-w)^{3}(i-w)^{2} w^{3}}, \\
& f_{3}(w)=\frac{p_{3}(w)}{q_{3}(w)}=\frac{p_{3}(w)}{(1-w)^{3}(i-w)^{3} w^{2}},
\end{aligned}
$$

with

$$
\left[p_{j}(w), q_{j}(w)\right]=1, \operatorname{deg} p_{j}(w) \leqq 7, \operatorname{deg} q_{j}(w)=8, j=1,2,3 .
$$

(d) Generalizations [3] of the Weierstrass formulas for the coordinate functions of a minimal surface in isothermal representation can be applied to obtain examples of rational and logarithmico-rational minimal surfaces in $E_{n}, n>3$. Thus, with

$$
(u, v)=w=(r \cos \theta, r \sin \theta),
$$

the formulas

$$
\begin{aligned}
& x_{1}(u, v)=12 r \cos \theta-3 r^{4} \cos 4 \theta, \\
& x_{2}(u, v)=-12 r \sin \theta-3 r^{4} \sin 4 \theta, \\
& x_{3}(u, v)=6 r^{2} \cos 2 \theta+4 r^{3} \cos 3 \theta, \\
& x_{4}(u, v)=-6 r^{2} \sin 2 \theta+4 r^{3} \sin 3 \theta
\end{aligned}
$$

represent an entire rational minimal surface, of degree 4, in Euclidean 4-space. This surface is not contained in any 3-dimensional Euclidean subspace. The surface has a pole of order 4 at $w=\infty$; it single zero is at $w=0$ and is of order 1 .

(e) The relations

$$
\begin{aligned}
& x_{1}=\Re\left(\log w-\frac{1}{2} w^{2}\right)=\log r-\frac{1}{2} r^{2} \cos 2 \theta, \\
& x_{2}=\Re\left(i \log w+\frac{i}{2} w^{2}\right)=-\theta-\frac{1}{2} r^{2} \sin 2 \theta, \\
& x_{3}=\Re(2 w)=2 r \cos \theta, \quad w=r(\cos \theta+i \sin \theta),
\end{aligned}
$$


give an isothermal representation of a minimal surface with a singularity of a different sort at the origin. The second of the relations (35) is not a (single-valued) function of $w=u+i v$, however, so that this surface is not included in the class of surfaces (1) presently under consideration.

The same remark might be made, of course, concerning the familiar relations

$$
\begin{aligned}
& x_{1}=\log r, \\
& x_{2}=\theta,
\end{aligned}
$$

which give an isothermal representation of a minimal surface in $E_{2}$, and for which $x_{1}+i x_{2}=\log w$.

6. The first fundamental theorem for meromorphic minimal surfaces. Nevanlinna's first fundamental theorem concerning meromorphic functions of a complex variable [15; 12, p. 212] can be generalized [7] to give an analogous result concerning meromorphic minimal surfaces. The concepts involved in the generalization are central to the discussion in the rest of the present paper.

In this section, some definitions are given and the first fundamental theorem and its generalization are stated without proof; but see $\S 8$ for the formulas from which these theorems result.

Let $f(w)$ be a nonconstant meromorphic function, and let $S$ be a nonconstant meromorphic minimal surface given in isothermal representation by (1) in the entire finite $w$-plane, $w=u+i v,|w|<\infty$.

In the classical Nevanlinna theory, for $0<r<\infty$ and for $a$ in the closed complex plane, the proximity function $m(r, a ; f)$ is defined by

$$
\begin{aligned}
& m(r, a ; f)=\frac{1}{2 \pi} \int_{0}^{2 \pi} \log ^{+} \frac{1}{\left|f\left(r e^{i \theta}\right)-a\right|} d \theta, \quad a \neq \infty, \\
& m(r, \infty ; f)=\frac{1}{2 \pi} \int_{0}^{2 \pi} \log ^{+}\left|f\left(r e^{i \theta}\right)\right| d \theta .
\end{aligned}
$$

By analogy, for $\boldsymbol{a}$ in the closed $n$-dimensional space, the proximity function $m(r, \boldsymbol{a} ; S)$ is defined by

$$
\begin{aligned}
m(r, \boldsymbol{a} ; S) & =\frac{1}{2 \pi} \int_{0}^{2 \pi} \log ^{+} \frac{1}{\left|\boldsymbol{x}\left(r e^{i \theta}\right)-\boldsymbol{a}\right|} d \theta, \quad \boldsymbol{a} \neq \infty, \\
m(r, \infty ; S) & =\frac{1}{2 \pi} \int_{0}^{2 \pi} \log ^{+}\left|\boldsymbol{x}\left(r e^{i \theta}\right)\right| d \theta,
\end{aligned}
$$

where, for simplicity, we have introduced the notation

$$
|\boldsymbol{v}|=(\boldsymbol{v} \cdot \boldsymbol{v})^{1 / 2}
$$


and

$$
\boldsymbol{x}\left(r e^{i \theta}\right)=\boldsymbol{x}(r \cos \theta, r \sin \theta) .
$$

In the Ahlfors-Shimizu version $[2,17 ; 12$, p. 216] of the Nevanlinna theory, the closed complex $\zeta=f(w)$ plane is projected stereographically onto the sphere of radius $1 / 2$ having center at

$$
\left(x_{1}, x_{2}, x_{3}\right)=(0,0,1 / 2) \text {. }
$$

Distances between points in the $\zeta$-plane are replaced with the chordal distances between corresponding points on the sphere, and the spherical proximity function $m^{\circ}(r, a ; f)$ is accordingly defined by

$$
\begin{aligned}
m^{\circ}(r, a ; f) & =\frac{1}{2 \pi} \int_{0}^{2 \pi} \log \frac{\left[1+\left|f\left(r e^{i \theta}\right)\right|^{2}\right]^{1 / 2}\left(1+|a|^{2}\right)^{1 / 2}}{\left|f\left(r e^{i \theta}\right)-a\right|} d \theta, \quad a \neq \infty, \\
m^{\circ}(r, \infty ; f) & =\frac{1}{2 \pi} \int_{0}^{2 \pi} \log \left[1+\left|f\left(r e^{i \theta}\right)\right|^{2}\right]^{1 / 2} d \theta .
\end{aligned}
$$

For $S$, similarly, the closed $n$-dimensional space is projected stereographically onto the $(n+1)$-dimensional hypersphere $\mathscr{S}$ of radius $1 / 2$ having center at

$$
\left(x_{1}, x_{2}, \cdots, x_{n}, x_{n+1}\right)=(0,0, \cdots, 0,1 / 2) .
$$

Distances between points in the $n$-dimensional space are replaced with the chordal distances between corresponding points on the hypersphere, and the hyperspherical proximity function $m^{\circ}(r, \boldsymbol{a} ; S)$ is accordingly defined by

$$
\begin{aligned}
m^{\circ}(r, \boldsymbol{a} ; S) & =\frac{1}{2 \pi} \int_{0}^{2-\pi} \log \frac{\left[1+\left|\boldsymbol{x}\left(r e^{i \theta}\right)\right|^{2}\right]^{1 / 2}\left(1+|\boldsymbol{a}|^{2}\right)^{1 / 2}}{\left|\boldsymbol{x}\left(r e^{i \theta}\right)-\boldsymbol{a}\right|} d \theta, \quad \boldsymbol{a} \neq \infty, \\
m^{\circ}(r, \infty ; S) & =\frac{1}{2 \pi} \int_{0}^{2 \pi} \log \left[1+\left|\boldsymbol{x}\left(r e^{i \theta}\right)\right|^{2}\right]^{1 / 2} d \theta .
\end{aligned}
$$

For $0 \leqq \rho<\infty$ and for $a$ in the closed complex plane, let $n(\rho, a ; f)$ denote the number of $a$-points of $f(w)$ in $|w| \leqq \rho$. Then the enumerative function $N(r, a ; f)$ is defined, for $0<r<\infty$, by

$$
N(r, a ; f)=\int_{0}^{r} \frac{n(\rho, a ; f)-n(0, a ; f)}{\rho} d \rho+n(0, a ; f) \log r,
$$

for a finite or infinite.

Similarly, for $\boldsymbol{a}$ in the closed $n$-dimensional space, and with an analogous definition of $n(\rho, \boldsymbol{a} ; S)$ as the number of $\boldsymbol{a}$-points of $S$ in $|w| \leqq \rho$, the enumerative function $N(r, \boldsymbol{a} ; S)$ is defined, for $0<r<$ $\infty$, by 
(38)

$$
N(r, \boldsymbol{a} ; S)=\int_{0}^{r} \frac{n(\rho, \boldsymbol{a} ; S)-n(0, \boldsymbol{\alpha} ; S)}{\rho} d \rho+n(0, \boldsymbol{a} ; S) \log r,
$$

for $\boldsymbol{a}$ finite or infinite.

For the extended theory, a function that does not occur in the classical theory is needed.

For any finite $a$, we have

$$
\Delta \log |f(w)-a|=0
$$

except at the isolated $a$-points and poles of $f(w)$, since

$$
\log |f(w)-a|=\Re \log [f(w)-a] .
$$

By contrast, for any finite $a$, a computation [8] using the fact that the $x_{j}(u, v)$ form an $n$-tuple of conjugate harmonic functions shows that

$$
\begin{aligned}
& \Delta \log |\boldsymbol{x}-\boldsymbol{a}| \\
= & \frac{2 \lambda\left\{|\boldsymbol{x}-\boldsymbol{a}|^{2}-\left[(\boldsymbol{x}-\boldsymbol{a}) \cdot(\partial \boldsymbol{x} / \partial u) / \lambda^{1 / 2}\right]^{2}-\left[(\boldsymbol{x}-\boldsymbol{a}) \cdot(\partial \boldsymbol{x} / \partial v) / \lambda^{1 / 2}\right]^{2}\right\}}{|\boldsymbol{x}-\boldsymbol{a}|^{4}}
\end{aligned}
$$

except at the isolated $\boldsymbol{a}$-points and poles of $S$ and the isolated zeros and infinities of $\lambda$.

Since $|\boldsymbol{x}(u, v)-\boldsymbol{a}|^{2}$ is the square of the length of the geometric vector $\boldsymbol{x}(u, v)-\boldsymbol{a}$, and

$$
\left\{[\boldsymbol{x}(u, v)-\boldsymbol{a}] \cdot \frac{\partial \boldsymbol{x}(u, v) / \partial u}{[\lambda(u, v)]^{1 / 2}}\right\}^{2}+\left\{[\boldsymbol{x}(u, v)-\boldsymbol{a}] \cdot \frac{\partial \boldsymbol{x}(u, v) / \partial v}{[\lambda(u, v)]^{1 / 2}}\right\}^{2}
$$

is the square of the length of the projection of $\boldsymbol{x}(u, v)-\boldsymbol{a}$ on the plane tangent to $S$ at $(u, v)$, it follows from (39) that

$$
\Delta \log |\boldsymbol{x}(u, v)-\boldsymbol{a}| \geqq 0
$$

at the nonexceptional points, ordinarily with the strict inequality for $n>2$.

The function

$$
\Delta \log \left|\boldsymbol{x}\left(r e^{i \theta}\right)-\boldsymbol{a}\right|
$$

is continuous except at the isolated $\boldsymbol{a}$-points and poles of $S$. A computation shows that, as $r \rightarrow 0,(41)$ is $O(1)$ at the $\boldsymbol{a}$-points and poles except at poles of order 1 with nonvanishing logarithmic part, where it is $O\left[(\log r)^{2}\right]$. Thus (41) can become infinite, but not rapidly enough to render it nonintegrable.

For $0 \leqq \rho<\infty$ and for any finite $\boldsymbol{a}$, the function $h(\rho, \boldsymbol{a} ; S)$ is defined by

$$
h(\rho, \boldsymbol{a} ; S)=\frac{1}{2 \pi} \iint_{i w \mid \leqq \rho} \Delta \log |\boldsymbol{x}(w)-\boldsymbol{a}| d A_{w} .
$$


In particular, we have

$$
h(0, \boldsymbol{a} ; S)=0 .
$$

The function $h(\rho, \boldsymbol{a} ; S)$ given by (42) might be considered to furnish a measure of the visibility of the map of $|w| \leqq \rho$ on $S$, as viewed from $a$.

Since

$$
\lim _{a \rightarrow \infty} h(\rho, \boldsymbol{a} ; S)=0,
$$

we define $h(\rho, \infty ; S)$ by

$$
h(\rho, \infty ; S)=0 \text {. }
$$

To the proximity function $m(r, \boldsymbol{a} ; S)$ or $m^{\circ}(r, \boldsymbol{a} ; S)$ and the enumerative function $N(r, \boldsymbol{a} ; S)$, we now adjoin a visibility function $H(r$, $a$; $S$ ), defined, for $0<r<\infty$, by

$$
H(r, \boldsymbol{a} ; S)=\int_{0}^{r} \frac{h(\rho, \boldsymbol{a} ; S)}{\rho} d \rho,
$$

for $\boldsymbol{a}$ finite or infinite. In particular, then, by (44), we have

$$
H(r, \infty ; S)=0 \text {. }
$$

By (43) and (44), the definition (45) of $H(r, \boldsymbol{a} ; S)$ is quite analogous to the definition (38) of $N(r, \boldsymbol{a} ; S)$, with $h(\rho, \boldsymbol{a} ; S)$ in place of $n(\rho, \boldsymbol{a} ; S)$.

In the classical Nevanlinna theory, the total affinity $\mathfrak{2}(r, a ; f)$ of $f(w)$ to $a$, or the affinity function of $f(w)$, is defined by

$$
\mathfrak{A}(r, a ; f)=m(r, a ; f)+N(r, a ; f)
$$

for $a$ finite or infinite. In particular, $\mathfrak{X}(r, \infty ; f)$ is called the Nevanlinna characteristic function of $f(w)$ and is denoted by $T(r ; f)$, so that

$$
T(r ; f)=\mathfrak{U}(r, \infty ; f)=m(r, \infty ; f)+N(r, \infty ; f) .
$$

The first fundamental theorem of Nevanlinna is the statement that, for each finite $a, T(r ; f)$ differs from $\mathfrak{X}(r, a ; f)$ by a bounded function of $r$ :

$$
T(r ; f)=\mathfrak{U}(r, a ; f)+C(r, a ; f) .
$$

In the extended theory, the total affinity $\mathfrak{2}(r, \boldsymbol{a} ; S)$ of $S$ to $\boldsymbol{a}$, or the affinity function of $S$, is defined by

$$
\mathfrak{N}(r, \boldsymbol{a} ; S)=m(r, \boldsymbol{a} ; S)+N(r, \boldsymbol{a} ; S)+H(r, \boldsymbol{a} ; S)
$$

for $\boldsymbol{a}$ finite or infinite. In particular, $\mathfrak{2}(r, \infty ; S)$ is called the Nevanlinna characteristic function of $S$ and is denoted by $T(r ; S)$, so that 


$$
\begin{aligned}
T(r ; S)=\mathfrak{U}(r, \infty ; S) & =m(r, \infty ; S)+N(r, \infty ; S)+H(r, \infty ; S) \\
& =m(r, \infty ; S)+N(r, \infty ; S) .
\end{aligned}
$$

The extended first fundamental theorem is then the statement that, for each finite $\boldsymbol{a}, T(r ; S)$ differs from $\mathfrak{N}(r, \boldsymbol{a} ; S)$ by a bounded function of $r$ :

$$
T(r ; S)=\mathfrak{U}(r, \boldsymbol{a} ; S)+C(r, \boldsymbol{a} ; S) .
$$

In the Ahlfors-Shimizu version of the classical theory, the proximity function is replaced by the spherical proximity function. The correction term then reduces to a constant for each finite $a$. It is customary in this case to add a constant to both sides of the equation so that each approaches 0 as $r \rightarrow 0$. Thus the spherical affinity function $\mathfrak{U}(r, a ; f)$ is defined by

$$
\mathfrak{H}^{\circ}(r, a ; f)=m^{\circ}(r, a ; f)+N(r, a ; f)+C(a ; f),
$$

for a finite or infinite, with the constant $C(a ; f)$ chosen so that

$$
\lim _{r \rightarrow 0} \mathfrak{N}^{\circ}(r, a ; f)=0 ;
$$

the spherical characteristic functions $T^{\circ}(r ; f)$ is defined by

$$
\begin{aligned}
T^{\circ}(r ; f) & =\mathfrak{H}^{\circ}(r, \infty ; f) \\
& =m^{\circ}(r, \infty ; f)+N(r, \infty ; f)+C(\infty ; f) ;
\end{aligned}
$$

and the spherical form of the first fundamental theorem is the statement that, for each finite a,

$$
T^{\circ}(r ; f)=\mathfrak{U}^{\circ}(r, a ; f) .
$$

Similarly, in the extension of the Ahlfors-Shimizu version, the hyperspherical affinity function $\mathfrak{U}^{\circ}(r, \boldsymbol{a} ; S)$ is defined by

$$
\mathfrak{X}^{\circ}(r, \boldsymbol{a} ; S)=m^{\circ}(r, \boldsymbol{a} ; S)+N(r, \boldsymbol{a} ; S)+H(r, \boldsymbol{a} ; S)+C(\boldsymbol{a} ; S),
$$

for $\boldsymbol{a}$ finite or infinite, with the constant $C(\boldsymbol{a} ; S)$ chosen so that

$$
\lim _{r \rightarrow 0} \mathfrak{X}^{\circ}(r, \boldsymbol{a} ; S)=0 ;
$$

the hyperspherical characteristic function $T^{\circ}(r ; S)$ is defined by

$$
\begin{aligned}
T^{\circ}(r ; S) & =\mathfrak{U}^{\circ}(r, \infty ; S) \\
& =m^{\circ}(r, \infty ; S)+N(r, \infty ; S)+H(r, \infty ; S)+C(\infty ; S) \\
& =m^{\circ}(r, \infty ; S)+N(r, \infty ; S)+C(\infty ; S) ;
\end{aligned}
$$

and the hyperspherical form of the first fundamental theorem is the statement that, for each finite $\boldsymbol{a}$, 


$$
T^{\circ}(r ; S)=\mathfrak{X}^{\circ}(r, \boldsymbol{a} ; S) .
$$

7. Basic growth properties. Proofs of the following convexity properties can be found in $[4,7]$.

Like the function $N(r, a ; f)$ in the complex-variable case, the function $N(r, \boldsymbol{a} ; S)$ for a meromorphic minimal surface $S$ is a nondecreasing, piecewise linear, convex function of $\log r$.

The function $H(r, \boldsymbol{a} ; S)$ vanishes identically if $\boldsymbol{a}$ is infinite or if $S$ is a plane surface and $\boldsymbol{a}$ lies in the plane. Otherwise, for $r>0$, $H(r, \boldsymbol{a} ; S)$ is a positive, increasing, strictly convex function of $\log r$.

The function $T^{\circ}(r ; S)$ can be given a quasi-geometric representation by integrating both members of (55) over the hyperspherical surface $\mathscr{S}$ and dividing by the content $\mathscr{C}$ of $\mathscr{S}$. After a simplification, this yields

$$
T^{\circ}(r ; S)=\int_{0}^{r} \mathscr{H}(\rho ; S) \frac{d \rho}{\rho}
$$

where

$$
\mathscr{H}(\rho ; S)=\frac{1}{\mathscr{Y}} \int \cdots \int_{\mathscr{S}} h(\rho, \boldsymbol{a} ; S) d \mathscr{Y}_{\boldsymbol{a}} .
$$

From (40), (42), (56), and (57), it follows that the function $T^{\circ}(r ; S)$ is positive for $r>0$ and is an increasing, strictly convex function of $\log r$.

From (56), we therefore obtain

$$
T^{\circ}(r ; S)>\mathscr{H}(1 ; S) \log r
$$

for $r>1$, and accordingly

$$
\liminf _{r \rightarrow \infty} \frac{T^{\circ}(r ; S)}{\log r}>0 .
$$

In $\$ \S 9$ and 10 of this paper, we shall be concerned with meromorphic minimal surfaces $S$ for which the left-hand member of (59) has a finite value.

We mention in passing, however, that just as the order $\rho(f)$ of a meromorphic function $f(w)$ is defined by

$$
\rho(f)=\limsup _{r \rightarrow \infty} \frac{\log T^{\circ}(r ; f)}{\log r},
$$

so is the order of a meromorphic minimal surface $S$, given in isothermal representation by (1), defined by

$$
\rho(S)=\limsup _{r \rightarrow \infty} \frac{\log T^{\circ}(r ; S)}{\log r} .
$$


Since for any two nonnegative numbers $a$ and $b$, we have

$$
\log ^{+}(a+b) \leqq \log ^{+} a+\log ^{+} b+\log 2,
$$

it follows from (36) and (37) that

$$
0 \leqq m(r, \infty ; S) \leqq m^{\circ}(r, \infty ; S) \leqq m(r, \sim ; S)+\log 2^{1 / 2} .
$$

Therefore, by (48) and (54), the difference $T^{\circ}(r ; S)-T(r ; S)$ is a bounded function of $r$. For this reason, by $(59), T^{\circ}(r ; S)$ and $T(r ; S)$ are interchangeable in the study of growth properties as $r \rightarrow \infty$.

Actually, it can be shown that, like $T^{\circ}(r ; S)$, the function $T(r ; S)$ is an increasing, strictly convex function of $\log r$.

8. The Poisson-Jensen-Nevanlinna formula. The first fundamental theorem of Nevanlinna, (47) or (51), results from the following Jensen-Nevanlinna formula [15].

Let $f(w)$ be meromorphic in $|w| \leqq R$, and in the neighborhood of the origin let

$$
f(w)=\alpha_{t} w^{t}+\alpha_{t+1} w^{t+1}+\cdots,
$$

with $\alpha_{t} \neq 0$, so that at the origin $f(w)$ has a zero of order $t$ if $t>0$ or a pole of order $-t$ if $t<0$, and $f(0) \neq 0$ or $\infty$ if $t=0$. Further, let the zeros and poles of $f(w)$ in $0<|w| \leqq R$ be at

$$
z_{1}, z_{2}, \cdots, z_{\mu} \text { and } p_{1}, p_{2}, \cdots, p_{\nu},
$$

respectively, with repetitions for multiple values and with

$$
0<\left|z_{1}\right| \leqq \cdots \leqq\left|z_{\mu}\right|<R \text { and } 0<\left|p_{1}\right| \leqq \cdots \leqq\left|p_{\nu}\right|<R .
$$

Then the Jensen-Nevanlinna formula is

$$
\begin{aligned}
\log \left|\alpha_{t}\right|= & \frac{1}{2 \pi} \int_{0}^{2 \pi} \log \left|f\left(R e^{i \theta}\right)\right| d \theta \\
& -\sum_{j=1}^{\mu} \log \frac{R}{\left|z_{j}\right|}+\sum_{k=1}^{\nu} \log \frac{R}{\left|p_{k}\right|}-t \log R .
\end{aligned}
$$

The extension, (50) or (55), of the first fundamental theorem to meromorphic minimal surfaces results similarly from an extension [7] of the Jensen-Nevanlinna formula.

Namely, for a meromorphic minimal surface $S$, given in isothermal representation by (1) for $|w| \leqq R, w=u+i v$, let the $z_{j}$ and $p_{k}$ have the same meaning as above but this time relative to $S$, and in the neighborhood of the origin let

$$
\boldsymbol{x}(u, v)=\boldsymbol{c} \log r+\sum_{k=t}^{\infty} r^{k}\left(\boldsymbol{a}_{k} \cos k \theta+\boldsymbol{b}_{k} \sin k \theta\right),
$$


with $\left|\boldsymbol{a}_{t}\right|^{2}+\left|\boldsymbol{b}_{t}\right|^{2} \neq 0$.

Then the extension of the Jensen-Nevanlinna formula is

$$
\begin{aligned}
\log \left|\boldsymbol{a}_{t}\right|= & \frac{1}{2 \pi} \int_{0}^{2 \pi} \log \left|\boldsymbol{x}\left(R e^{i \theta}\right)\right| d \theta \\
& -\sum_{j=1}^{\mu} \log \frac{R}{\left|\boldsymbol{z}_{j}\right|}+\sum_{k=1}^{\nu} \log \frac{R}{\left|p_{k}\right|}-t \log R \\
& -\frac{1}{2 \pi} \int_{0}^{R}\left[\frac{1}{\rho} \iint_{|w| \leqq \rho} \Delta \log |\boldsymbol{x}(w)| d A_{w}\right] d \rho .
\end{aligned}
$$

An important tool in the further investigation of meromorphic functions $f(w)$ is obtained by mapping the circular disc $|w| \leqq R$ conformally onto itself, with a given point

$$
w_{0}=u_{0}+i v_{0}=r e^{i \varphi}, \quad r<R,
$$

mapped onto the origin. If $f\left(w_{0}\right) \neq 0$ or $\infty$, and if the symbols $z_{j}$ and $p_{k}$ now represent all the zeros and poles in the disc $|w| \leqq R$, not merely those in the punctured disc $0<|w| \leqq R$, then an application of the Jensen-Nevanlinna formula (63) in the transformed disc yields the Poisson-Jensen-Nevanlinna formula,

$$
\begin{aligned}
\log \left|f\left(w_{0}\right)\right|= & \frac{1}{2 \pi} \int_{0}^{2 \pi} \log \left|f\left(R e^{i \theta}\right)\right| \frac{R^{2}-r^{2}}{R^{2}-2 R r \cos (\theta-\varphi)+r^{2}} d \theta \\
& -\sum_{j=1}^{\mu} \log \left|\frac{R^{2}-\bar{z}_{j} w_{0}}{R\left(w_{0}-a_{j}\right)}\right|+\sum_{k=1}^{\nu} \log \left|\frac{R^{2}-\bar{p}_{k} w_{0}}{R\left(w_{0}-p_{k}\right)}\right| .
\end{aligned}
$$

In the same way, an application of the extension (65) of the Jensen-Nevanlinna formula in the transformed disc yields an extension of the Poisson-Jensen-Nevanlinna formula to meromorphic minimal surfaces $S$. Namely, with the same notation as above, but now relative to $S$, the extension of the Poisson-Jensen-Nevanlinna formula is

$$
\begin{aligned}
\log \left|\boldsymbol{x}\left(w_{0}\right)\right| & =\frac{1}{2 \pi} \int_{0}^{2 \pi} \log \left|\boldsymbol{x}\left(R e^{i \theta}\right)\right| \frac{R^{2}-r^{2}}{R^{2}-2 R r \cos (\theta-\varphi)+r^{2}} d \theta \\
& -\sum_{j=1}^{n} \log \left|\frac{R^{2}-\bar{z}_{j} w_{0}}{R\left(w_{0}-z_{j}\right)}\right|+\sum_{k=1}^{\nu} \log \left|\frac{R^{2}-\bar{p}_{k} w_{0}}{R\left(w_{0}-p_{k}\right)}\right| \\
& -\frac{1}{2 \pi} \int_{0}^{R}\left[\frac{1}{\rho} \iint_{B\left(\rho, w_{0}\right)} \Delta \log |\boldsymbol{x}(w)| d A_{w}\right] d \rho,
\end{aligned}
$$

where

$$
B\left(\rho, w_{0}\right)=\left\{w:\left|\frac{R^{2}\left(w-w_{0}\right)}{R^{2}-\bar{w}_{0} w}\right| \leqq \rho\right\} .
$$

[Like the Jensen-Nevanlinna formula (63) and its extension (65), the Poisson-Jensen-Nevanlinna formula (67) and its extension (68) can 
readily be adjusted to include the case in which there is a zero or pole at $w_{0}$.]

Notice in (68) that

$$
\sum_{j=1}^{\mu} \log \left|\frac{R^{2}-\bar{z}_{j} w_{0}}{R\left(w_{0} z_{j}\right)}\right| \geqq 0 \quad \text { and } \quad \sum_{k=1}^{\nu} \log \left|\frac{R^{2}-p_{k} w_{0}}{R\left(w_{0}-p_{k}\right)}\right| \geqq 0,
$$

and that also, by (40),

$$
\frac{1}{2 \pi} \int_{0}^{R}\left[\frac{1}{\rho} \iint_{B\left(\rho, w_{0}\right)} \Delta \log |\boldsymbol{x}(w)| d A_{w}\right] d \rho \geqq 0 .
$$

Substituting from (71) and the first inequality in (70) into (68), we obtain the useful inequality

$$
\begin{aligned}
\log \left|\boldsymbol{x}\left(w_{0}\right)\right| \leqq & \frac{1}{2 \pi} \int_{0}^{2 \pi} \log \left|\boldsymbol{x}\left(R e^{i \theta}\right)\right| \frac{R^{2}-r^{2}}{R^{2}-2 R r \cos (\theta-\varphi)+r^{2}} d \theta \\
& +\sum_{k=1}^{\nu} \log \left|\frac{R^{2}-\bar{p}_{k} w_{0}}{R\left(w_{0}-p_{k}\right)}\right| .
\end{aligned}
$$

A fortiori, we therefore have

$$
\begin{aligned}
\log ^{+}\left|\boldsymbol{x}\left(w_{0}\right)\right| \leqq & \frac{1}{2 \pi} \int_{0}^{2 \pi} \log ^{+}\left|\boldsymbol{x}\left(R e^{i \theta}\right)\right| \frac{R^{2}-r^{2}}{R^{2}-2 R r \cos (\theta-\varphi)+r^{2}} d \theta \\
& +\sum_{k=1}^{\nu} \log \left|\frac{R^{2}-\bar{p}_{k} w_{0}}{R\left(w_{0}-p_{k}\right)}\right|,
\end{aligned}
$$

and accordingly, by (36),

$$
\log ^{+}\left|\boldsymbol{x}\left(w_{0}\right)\right| \leqq \frac{R+r}{R-r} m(R, \infty ; S)+\sum_{k=1}^{\nu} \log \left|\frac{R^{2}-\bar{p}_{k} w_{0}}{R\left(w_{0}-p_{k}\right)}\right| .
$$

In particular, letting

$$
M(r ;|\boldsymbol{x}|)=\sup _{0 \leqq \varphi<2 \pi}\left|\boldsymbol{x}\left(r e^{i \varphi}\right)\right|,
$$

and choosing $w_{r}^{*}=r e^{i \varphi *}$ so that

$$
\left|\boldsymbol{x}\left(w_{r}^{*}\right)\right|=M(r ;|\boldsymbol{x}|),
$$

from (74) we obtain

$$
\log ^{+} M(r ;|\boldsymbol{x}|) \leqq \frac{R+r}{R-r} m(R, \infty ; S)+\sum_{k=1}^{\nu} \log \left|\frac{R^{2}-\bar{p}_{k} w_{r}^{*}}{R\left(w_{r}^{*}-p_{k}\right)}\right| .
$$

9. An alternative characterization of rational and logarithmico-rational minimal surfaces. We shall now apply (77) in the study of meromorphic minimal surfaces for which the left-hand member of (59) has a finite value. 
THEOREM 2. For a nonconstant meromorphic minimal surface $S$ given in isothermal representation by $(1), T^{\circ}(r ; S)$ satisfies

$$
\liminf _{r \rightarrow \infty} \frac{T^{\circ}(r ; S)}{\log r}=c<\infty
$$

if and only if $c$ is a positive integer and $S$ is a rational or logarithmicorational minimal surface of degree $c$.

Proof. Suppose first that (78) is satisfied. It follows then from (54) that

$$
\liminf _{r \rightarrow \infty} \frac{N(r, \infty ; S)}{\log r} \leqq c,
$$

since $C(\infty ; S)$ is a constant and since, by $(37), m^{\circ}(r, \infty ; S)$ is positive for $r>0$.

Therefore, by (38) and (79), $S$ can have at most a finite number of poles, and accordingly the vector function $\boldsymbol{x}(u, v)$ in (1) has a representation of the form (25).

We shall see now that (78) implies further that the representation (25) reduces to $(26)$.

Let $r_{0}$ be great enough that all the poles of $S$ in the finite plane are in $|w|<r_{0}$. Then for $r>r_{0}$ and $R=\delta r$, with $\delta>1$ fixed, and with the notation of (25), (77) gives

$$
\begin{aligned}
\log ^{+}|M(r ;|\boldsymbol{x}|)| & \leqq \frac{\delta+1}{\delta-1} m(\delta r, \infty ; S)+\sum_{j=1}^{l} \log \left|\frac{\delta^{2} r^{2}-\bar{w}_{j} w_{r}^{*}}{\delta r\left(w_{r}^{*}-w_{j}\right)}\right|^{n_{j}} \\
& \leqq \frac{\delta+1}{\delta-1} m(\delta r, \propto ; S)+O(1)
\end{aligned}
$$

as $r \rightarrow \infty$, since the logarithmic sum is a bounded function of $r$.

From (80), we obtain

$$
\begin{aligned}
\liminf _{r \rightarrow \infty} \frac{\log ^{+} M(r ;|\boldsymbol{x}|)}{\log r} & \leqq \frac{\delta+1}{\delta-1} \liminf _{r \rightarrow \infty}\left[\frac{m(\delta r, \infty ; S)}{\log \delta r} \cdot \frac{\log \delta+\log r}{\log r}\right] \\
& =\frac{\delta+1}{\delta-1} \liminf _{r \rightarrow \infty} \frac{m(r, \infty ; S)}{\log r} .
\end{aligned}
$$

Letting $\delta \rightarrow \infty$ now yields

$$
\liminf _{r \rightarrow \infty} \frac{\log ^{+} M(r ;|\boldsymbol{x}|)}{\log r} \leqq \liminf _{r \rightarrow \infty} \frac{m(r, \infty ; S)}{\log r} .
$$

Therefore, by (54), (62), and (78),

$$
\liminf _{r \rightarrow \infty} \frac{\log ^{+} M(r ;|\boldsymbol{x}|)}{\log r} \leqq c,
$$


since $C(\infty ; S)$ is a constant and since, by $(38), N(r, \propto ; S)$ is nonnegative for $r \geqq 1$.

By (21), (22), and (23), we have

$$
M(r ;|\boldsymbol{y}|) \leqq M(r ;|\boldsymbol{x}|)+O(\log r)
$$

as $r \rightarrow \infty$, and therefore

$$
\begin{aligned}
\liminf _{r \rightarrow \infty} \frac{M(r ;|\boldsymbol{y}|)}{\log r} & \leqq \liminf _{r \rightarrow \infty} \frac{\log [M(r ;|\boldsymbol{x}|)+O(\log r)]}{\log r} \\
& =\liminf \frac{\log M(r ;|\boldsymbol{x}|)}{\log r} \leqq c .
\end{aligned}
$$

Hence for every $\varepsilon>0$ there is a sequence $\left\{r_{k}\right\}$, with $r_{k} \rightarrow \infty$, for which

$$
\lim _{k \rightarrow \infty} M\left(r_{k} ;|\boldsymbol{y}|\right)=L<r^{c+\varepsilon}
$$

But from (24) we obtain

$$
\frac{1}{2 \pi} \int_{0}^{2 \pi}\left|\boldsymbol{y}\left(r e^{i \theta}\right)\right|^{2} d \theta=\sum_{k=0}^{\infty}\left(\left|\boldsymbol{a}_{k}\right|^{2}+\left|\boldsymbol{b}_{k}\right|^{2}\right) r^{2 k} .
$$

Now (84) and (85) imply that there is a $k_{0}<\infty$ such that for all $k>k_{0}$ we have

$$
\left|\boldsymbol{a}_{k}\right|^{2}+\left|\boldsymbol{b}_{k}\right|^{2}=0 \text {. }
$$

Thus $\boldsymbol{y}(u, v)$ is a polynomial and therefore (25) reduces to the form (26); that is, the nonconstant meromorphic minimal surface $S$ is a rational or logarithmico-rational minimal surface of degree $m>0$.

We shall now show that the constant $c$ in (78) is a positive integer by showing that $c=m$, and shall at the same time prove the second part of the theorem. We shall in fact prove more:

If $S$ is a rational or logarithmico-rational minimal surface of degree $m>0$, then

$$
\lim _{r \rightarrow \infty} \frac{T^{\circ}(r ; S)}{\log r}
$$

exists, and

$$
\lim _{r \rightarrow \infty} \frac{T^{\circ}(r ; S)}{\log r}=m
$$

With $S$ in the representation (26), and with $m$ given by (32), by (38) we have

$$
\lim _{r \rightarrow \infty} \frac{N(r, \infty ; S)}{\log r}=\sum_{j=1}^{l} n_{j}
$$


We now need an improvement of (82). Since, by (36) and (75), we have

$$
\log ^{+} M(r ;|\boldsymbol{x}|) \geqq m(r, \infty ; S),
$$

it follows that (82) holds also with the inequality sign reversed, and therefore that

$$
\liminf _{r \rightarrow \infty} \frac{\log ^{+} M(r ;|\boldsymbol{x}|)}{\log r}=\liminf _{r \rightarrow \infty} \frac{m(r, \infty ; S)}{\log r} .
$$

In the same way, with

$$
\limsup _{r \rightarrow \infty} \text { in place of } \liminf _{r \rightarrow \infty}
$$

in (81) and (82), from (88) we obtain

$$
\limsup _{r \rightarrow \infty} \frac{\log ^{+} M(r ;|\boldsymbol{x}|)}{\log r}=\limsup _{r \rightarrow \infty} \frac{m(r, \infty ; S)}{\log r} .
$$

By (26), however, we have

$$
\lim _{r \rightarrow \infty} \frac{\log ^{+} M(r ;|\boldsymbol{x}|)}{\log r}=n_{\infty}
$$

Therefore, by (89), (90), and (91),

$$
\lim _{r \rightarrow \infty} \frac{m(r, \infty ; S)}{\log r}=n_{\infty} .
$$

Thus, from (49), (87), (92), and a remark near the end of $\S 7$, we have

as desired.

$$
\begin{aligned}
\lim _{r \rightarrow \infty} \frac{T^{\circ}(r ; S)}{\log r} & =\lim _{r \rightarrow \infty} \frac{T(r ; S)}{\log r} \\
& =\lim _{r \rightarrow \infty} \frac{m(r, \infty ; S)}{\log r}+\lim _{r \rightarrow \infty} \frac{N(r, \propto ; S)}{\log r} \\
& =n_{\infty}+\sum_{j=1}^{l} n_{j}=m,
\end{aligned}
$$

10. The fundamental theorem of algebra. If $S$ is a rational or logarithmico-rational minimal surface of degree $m>0$, given in the representation (26), then the vector function $\boldsymbol{x}(u, v)$ maps every point of the closed $w$, or $u+i v$, plane onto a unique point $\boldsymbol{a}$, finite or infinite.

Let $n_{a} \geqq 0$ be the order of the $a$-point of $S$ at $w=\infty$ (of course, $n_{a}=0$ for all points $a$ except one), and let

$$
n(\boldsymbol{a} ; S)=n_{\boldsymbol{a}}+\lim _{r \rightarrow \infty} n(r, \boldsymbol{a} ; S),
$$




$$
h(\boldsymbol{a} ; S)=\lim _{r \rightarrow \infty} h(r, \boldsymbol{a} ; S) .
$$

Then $n(\boldsymbol{a} ; S)$ is the total number of $\boldsymbol{a}$-points, counting multiplicities, of $S$ in the closed $w$-plane, and $h(\boldsymbol{a} ; S)$ is a measure of the visibility of the entire surface $S$ when viewed from $a$.

In particular, for $\boldsymbol{a}=\propto$, by (26) and (32) we have

$$
n(\infty ; S)=n_{\infty}+\sum_{j=1}^{l} n_{j}=m,
$$

and by (44) we have

$$
h(\infty ; S)=0,
$$

so that

$$
n(\infty ; S)+h(\propto ; S)=m .
$$

We shall show now that (95) holds not only for $\infty$ but also for every finite $\boldsymbol{a}$; that is, we shall show that

$$
n(\boldsymbol{a} ; S)+h(\boldsymbol{a} ; S)=m .
$$

Let $\boldsymbol{a}$ be any finite point. By (37):

(i) If $n_{a}=0$ and $S$ has a pole at $\infty$, then

$$
m^{\circ}(r, \boldsymbol{a} ; S)=\log \left(1+|\boldsymbol{a}|^{2}\right)+O(1) .
$$

(ii) If $n_{\boldsymbol{a}}=0$ and $S$ has $\boldsymbol{a} \boldsymbol{b}$-point, $\boldsymbol{b} \neq \boldsymbol{a}$, at $\infty$, then

$$
m^{\circ}(r, \boldsymbol{a} ; S)=\log \frac{\left(1+|\boldsymbol{b}|^{2}\right)^{1 / 2}\left(1+|\boldsymbol{a}|^{2}\right)^{1 / 2}}{|\boldsymbol{b}-\boldsymbol{a}|}+O(1) .
$$

(iii) If $n_{a}>0$, then

$$
m^{\circ}(r, \boldsymbol{a} ; S)=\log r^{n} a+O(1) .
$$

In each of the three cases, we have

$$
\lim _{r \rightarrow \infty} \frac{m^{\circ}(r, \boldsymbol{a} ; S)}{\log r}=n_{a},
$$

since $n_{a}=0$ in the first two cases.

By (52), (55), (86), and (97), we therefore have

$$
\lim _{r \rightarrow \infty} \frac{N(r, \boldsymbol{a} ; S)+H(r, \boldsymbol{a} ; S)}{\log r}=m-n_{a} .
$$

We shall now show that

$$
\lim _{r \rightarrow \infty}[n(r, \boldsymbol{a} ; S)+h(r, \boldsymbol{a} ; S)]=m-n_{a} .
$$


Since $n(r, \boldsymbol{a} ; S)$ and $h(r, \boldsymbol{a} ; S)$ are nondecreasing functions of $r$, if (99) did not hold then either there would be an $\varepsilon>0$ such that

$$
n(r, \boldsymbol{a} ; S)+h(r, \boldsymbol{a} ; S) \leqq m-n_{\boldsymbol{a}}-\varepsilon
$$

for all $r$, or there would be an $\varepsilon>0$ and an $r_{0}$ such that

$$
n(r, \boldsymbol{a} ; S)+h(r, \boldsymbol{a} ; S) \geqq m-n_{\boldsymbol{a}}+\varepsilon
$$

for all $r \geqq r_{0}$. In the former case, by (38) and (45), we would have

$$
\varlimsup_{r \rightarrow \infty} \frac{N(r, \boldsymbol{a} ; S)+H(r, \boldsymbol{a} ; S)}{\log r} \leqq m-n_{a}-\varepsilon,
$$

and similarly in the latter case we would have

$$
\varliminf_{r \rightarrow \infty} \frac{N(r, \boldsymbol{a} ; S)+H(r, \boldsymbol{a} ; S)}{\log r} \geqq m-n_{a}+\varepsilon .
$$

Hence (99) follows from (98).

From (93), (94), and (99), we obtain

$$
\begin{aligned}
n(\boldsymbol{a} ; S)+h(\boldsymbol{a} ; S) & =n_{a}+\lim _{r \rightarrow \infty}[n(r, \boldsymbol{\alpha} ; S)+h(r, \boldsymbol{a} ; S)] \\
& =n_{a}+\left(m-n_{a}\right)=m .
\end{aligned}
$$

We have thus proved the following result:

THEOREM 3. If $S$ is a rational or logarithmico-rational minimal surface of degree $m>0$ in the extended $E_{n}$-space, then for each $\boldsymbol{a}$, including $\boldsymbol{a}=\infty$, in this space,

$$
n(\boldsymbol{a} ; S)+h(\boldsymbol{a} ; S)=m .
$$

Since $m$ is a positive integer, since $n(\boldsymbol{a} ; S)$ is a nonnegative integer and $h(\boldsymbol{a} ; S)$ is nonnegative, and since $h(\boldsymbol{a} ; S)=0$ if and only if either $\boldsymbol{a}=\infty$ or $S$ is a plane surface with $\boldsymbol{a}$ on $S$, it follows from (100) that:

(i ) For each $\boldsymbol{a}$, both $n(\boldsymbol{a} ; S)$ and $h(\boldsymbol{a} ; S)$ are nonnegative integers.

(ii) $n(\boldsymbol{a} ; S) \leqq m$, with equality if and only if either $\boldsymbol{a}=\infty$ or $S$ is a plane with $\boldsymbol{a}$ on $S$.

(iii) $h(\boldsymbol{a} ; S) \leqq m$, with equality for almost all points of $E_{n}$ if $n>2$.

(iv) Theorem 3 includes the fundamental theorem of algebra as a special case.

Since many proofs of the fundamental theorem of algebra are known, alternative proofs of Theorem 3 might also be desirable. In particular, $h(\boldsymbol{a} ; s)$ will shortly be interpreted, at least for $n=3$, in terms of areas on a sphere with center at $\boldsymbol{a}$, and this well might lead to a topological proof of the theorem. 


\section{REFERENCES}

1. V. Ahlfors, Complex Analysis, 2nd ed., McGraw-Hill Book Company, New York, 1966.

2. L. V. Ahlfors, Beiträge der meromorphen Funktionen, 7 Congr. Math. Scand., Oslo, 1929 .

3. E. F. Beckenbach, Minimal surfaces in Euclidean n-space, Amer. J. Math., 55 (1933), 458-468.

4. —, An introduction to the theory of meromorphic minimal surfaces, Proceedings of Symposia in Pure Mathematics, Vol. 11, Entire Functions and Related Parts of Analysis, American Mathematical Society, Providence, R. I., 1968.

5. — Defect relations for meromorphic minimal surfaces: an introduction, Colloquium on Mathematical Analysis, Jyvaskyla, Finland, 1970 (to appear).

6. E. F. Beckenbach and J. W. Hahn, Triples of conjugate harmonic functions and minimal surfaces, Duke Math. J., 2 (1936), 698-704.

7. E. F. Beckenbach and G. A. Hutchison, Meromorphic minimal surfaces, Pacific J. Math., 28 (1969), 17-47.

8. E. F. Beckenbach and T. Radó, Subharmonic functions and minimal surfaces, Trans. Amer. Math. Soc., 35 (1933), 648-661.

9. Henri Cartan, Elementary Theory of Analytic Functions of One or Several Complex Variables, Addison-Wesley Publishing Company, Inc., Palo Alto, 1963.

10. L. R. Ford, Automorphic Functions, McGraw-Hill Book Company, New York, 1929.

11. Einar Hille, Analytic Function Theory, Vol. 1, Ginn and Company, Boston, 1959.

12. - Analytic Function Theory, Vol. 2, Ginn and Company, Boston, 1962.

13. Barrett O'Neill, Elementary Differential Geometry, Academic Press, New York, 1966.

14. W. F. Osgood, Lehrbuch der Funktionentheorie, Vol. 1, B. G. Teubner, Leipzig, 1928.

15. R. Nevanlinna, Zur Theorie der meromorphen Funktionen, Acta Math., 46 (1925), 1-99.

16. Tibor Radó, On the Problem of Plateau, Julius Springer Verlag, Berlin, 1933.

17. T. Shimizu, On the theory of meromorphic functions, Japan J. Math., 6 (1929), $119-171$.

Received October 19, 1972. This work was supported in part under grant No. NSF GP 28971.

University of California, Los ANGeles

McDonnell Douglas Corporation

AND

Hughes Aircraft Company 



\section{PACIFIC JOURNAL OF MATHEMATICS}

\section{EDITORS}

RICHARD ARENS (Managing Editor)

University of California

Los Angeles, California 90024

\section{R. A. Beaumont \\ University of Washington \\ Seattle, Washington 98105}

\section{J. DugundjI*}

Department of Mathematics University of Southern California Los Angeles, California 90007

D. Gilbarg and J. Milgram

Stanford University

Stanford, California 94305

\section{ASSOCIATE EDITORS}
E. F. BeCKenBaCH
B. H. NeumanN
F. WOLF
K. YoSHIDA

\section{SUPPORTING INSTITUTIONS}

\author{
UNIVERSITY OF BRITISH COLUMBIA \\ CALIFORNIA INSTITUTE OF TECHNOLOGY \\ UNIVERSITY OF CALIFORNIA \\ MONTANA STATE UNIVERSITY \\ UNIVERSITY OF NEVADA \\ NEW MEXICO STATE UNIVERSITY \\ OREGON STATE UNIVERSITY \\ UNIVERSITY OF OREGON \\ OSAKA UNIVERSITY
}

\author{
UNIVERSITY OF SOUTHERN CALIFORNIA \\ STANFORD UNIVERSITY \\ UNIVERSITY OF TOKYO \\ UNIVERSITY OF UTAH \\ WASHINGTON STATE UNIVERSITY \\ UNIVERSITY OF WASHINGTON

$* * *$
$*$
AMERICAN MATHEMATICAL SOCIETY
NAVAL WEAPONS CENTER

The Supporting Institutions listed above contribute to the cost of publication of this Journal, but they are not owners or publishers and have no responsibility for its content or policies.

Mathematical papers intended for publication in the Pacific Journal of Mathematics should be in typed form or offset-reproduced, (not dittoed), double spaced with large margins. Underline Greek letters in red, German in green, and script in blue. The first paragraph or two must be capable of being used separately as a synopsis of the entire paper. Items of the bibliography should not be cited there unless absolutely necessary, in which case they must be identified by author and Journal, rather than by item number. Manuscripts, in duplicate if possible, may be sent to any one of the four editors. Please classify according to the scheme of Math. Rev. Index to Vol. 39. All other communications to the editors should be addressed to the managing editor, or Elaine Barth, University of California, Los Angeles, California, 90024.

100 reprints are provided free for each article, only if page charges have been substantially paid. Additional copies may be obtained at cost in multiples of 50 .

The Pacific Journal of Mathematics is issued monthly as of January 1966. Regular subscription rate: $\$ 60.00$ a year (6 Vols., 12 issues). Special rate: $\$ 30.00$ a year to individual members of supporting institutions.

Subscriptions, orders for back numbers, and changes of address should be sent to Pacific Journal of Mathematics, 103 Highland Boulevard, Berkeley, California, 94708.

PUBLISHED BY PACIFIC JOURNAL OF MATHEMATICS, A NON-PROFIT CORPORATION

Printed at Kokusai Bunken Insatsusha (International Academic Printing Co., Ltd.), 270, 3-chome Totsuka-cho, Shinjuku-ku, Tokyo 160, Japan

* C. R. DePrima California Institute of Technology, Pasadena, CA 91109, will replace J. Dugundji until August 1974. 


\section{Pacific Journal of Mathematics}

Vol. 50, No. $2 \quad$ October, 1974

Mustafa Agah Akcoglu, John Philip Huneke and Hermann Rost, A counter example to the Blum Hanson theorem in general spaces .............

Huzihiro Araki, Some properties of modular conjugation operator of von

Neumann algebras and a non-commutative Radon-Nikodym theorem

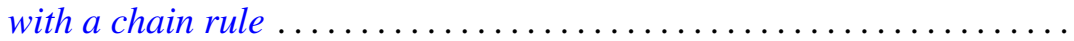

E. F. Beckenbach, Fook H. Eng and Richard Edward Tafel, Global properties of rational and logarithmico-rational minimal surfaces .....

David W. Boyd, A new class of infinite sphere packings ............. 383

K. G. Choo, Whitehead Groups of twisted free associative algebras ........

Charles Kam-Tai Chui and Milton N. Parnes, Limit sets of power series

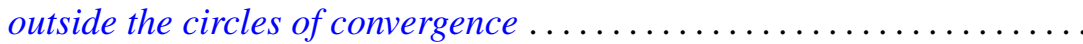

Allan Clark and John Harwood Ewing, The realization of polynomial

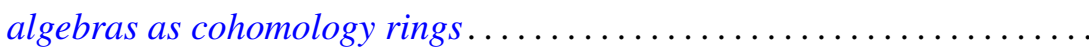

Dennis Garbanati, Classes of circulants over the p-adic and rational

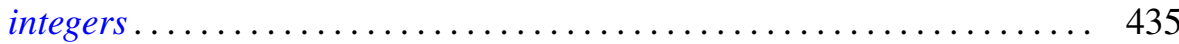

Arjun K. Gupta, On a "square" functional equation ................... 449

David James Hallenbeck and Thomas Harold MacGregor, Subordination and extreme-point theory ............................. 455

Douglas Harris, The local compactness of $v X \ldots \ldots . . . . . . . . . . . .4469$

William Emery Haver, Monotone mappings of a two-disk onto itself which fix the disk's boundary can be canonically approximated by homeomorphisms .................................. 477

Norman Peter Herzberg, On a problem of Hurwitz .................. 485

Chin-Shui Hsu, A class of Abelian groups closed under direct limits and subgroups formation ............................... 495

Bjarni Jónsson and Thomas Paul Whaley, Congruence relations and multiplicity types of algebras.....................

Lowell Duane Loveland, Vertically countable spheres and their wild sets.

Nimrod Megiddo, Kernels of compound games with simple components ....

Russell L. Merris, An identity for matrix functions ........ . .

E. O. Milton, Fourier transforms of odd and even tempered distributions ...

Dix Hayes Pettey, One-one-mappings onto locally connected generalized continua

Mark Bernard Ramras, Orders with finite global dimension

Doron Ravdin, Various types of local homogeneity. .

George Michael Reed, On metrizability of complete Moore spaces ...

Charles Small, Normal bases for quadratic extensions ..

Philip C. Tonne, Polynomials and Hausdorff matrices.... . . 